\title{
Simulating Urban Growth in Urumqi, Using the SLEUTH Model
}

\author{
Ya-Xuan Liu \\ School of Economics, Xinjiang University of Finance and Economics, Urumqi 830012, China \\ lyx1022@163.com
}

Keywords: Urban growth, Sleuth, Urumqi.

\begin{abstract}
Urumqi is the capital city of Xinjiang Uyghur Autonomous Region, in the northwestern part of China. The city has witnessed rapid growth in recent years, mostly because of the national and regional policies and its economic and social development. We implemented this study to understand the urban growth of Urumqi City, to forecast its growth tendency for the next two decades and to provide a basis for urban management. The SLEUTH are employed to build the urban growth simulation and forecasting model. We calibrated it with historical data derived from a time series of satellite images. The results showed the utility of the modeling method in explaining the spatial pattern of urban growth. In the future years during the forecast period, Urumqi city should focus on enforcing the urban internal spatial structure optimization and spatial displacement, paying attention to the urban internal differentiation, improving the economic value and utilization of urban land resources, controlling the speed of urban growth, and retaining certain land reserve and development space for the future development.
\end{abstract}

\section{Introduction}

Urban growth both in population and spatial extent has led to alterations of the landscape [1]. In the last 200 years, while the world population has increased six times, the urban population has multiplied 100 times [2]. As a developing country, China is now witnessing an almost continual large-scale urbanization. It is also occurring in western big cities such as Urumqi. This city is located in the arid and semiarid areas. After three hundred years of development, here gradually formed the oasis ecosystem and the unique urban landscape consists of farmland, forest, roads, residential points, channels, reservoirs and pasture. It has become a megacity in the center of the Eurasian continent. As a central city of international thoroughfare in western China, the city will enter the large-scale development and construction of the new period, with the deepening of China's western development strategy implementation and the economic and social development. With this rapid growth, cities exert a heavy pressure on lands and resources at their periphery [3]. In the decision-making process, land managers need to carefully consider the changes brought about by urban sprawl.

This study investigated the value of the combination of spatial modeling, remote sensing, and analysis with spatial metrics for Urumqi urban region between 1975 and 2030. The historical analysis was based on remote sensing observations from archived Landsat MSS, TM and ETM satellite data. Among all documented dynamic models, cellular automata (CA) probably are the most impressive in urban growth modeling in terms of their flexibility, their simplicity in application, their close ties to remote sensing data and geographic information system [4]. The remote sensing-derived data layers were used in parameterization and calibration of the SLEUTH urban growth model, which is one of the CA model [5]. The SLEUTH model was used to predict Urumqi's urban growth from 2010 to 2030, and to recreate missing historical time periods in the evolution of Urumqi's urban extent since 1975. A set of spatial metrics was used in a detailed analysis of the growth patterns mapped from the remote sensing data and were extended to interpret and analyze the model's forecasted growth in the area up to 2030.This paper shows a way of utilizing SLEUTH as an urban growth model, and interpret and analyze the spatial pattern of Urumqi's future growth reflected in the spatial metrics as applied to forecasts to the year 2030. This analysis is presented at the urban level, rather than the global level in order to clearly identify the mechanisms of urban change at the spatial scale of the modeling, especially in Chinese northwestern area. 


\section{Materials and methods}

\section{Study Area}

Urumqi City is the capital of Xinjiang Uyghur Autonomous Region and the political, economic, cultural, educational, scientific and technological center of the region. It is located in Urumqi River valley and the downstream alluvial-and-diluvial plain from $86^{\circ} 37^{\prime} 33^{\prime \prime E}$ to $88^{\circ} 58^{\prime} 24^{\prime \prime} \mathrm{E}$, and $42^{\circ} 45^{\prime} 32^{\prime \prime} \mathrm{N}$ to $44^{\circ} 08^{\prime} 32^{\prime \prime} \mathrm{N}$, where there is a natural barrier of city construction on both sides of the hills and mountains. The downtown is located in the middle and lower reaches of the Urumqi River valley, facing the vast alluvial plain in its north direction (Fig. 1). The urban planning control area is $1600 \mathrm{~km}^{2}$. At the end of 2013, the permanent population reaches 3.46 million. The GDP in this year is 240 billion yuan, and the GDP per capita is 69.4 thousand yuan which is in the front rank among the other provincial capitals and at the top of the 5 northwestern provincial capitals. After 1990, especially after the West Development Strategy has been carried out in 2000, urban growth developed at an unprecedented level in Urumqi. The built area grows from $49.00 \mathrm{~km}^{2}$ in 1985 to $391.20 \mathrm{~km}^{2}$ in 2013 , with an annual growth rate of $7.70 \%$, far ahead of the cities in other provinces and autonomous regions in Northwest China.

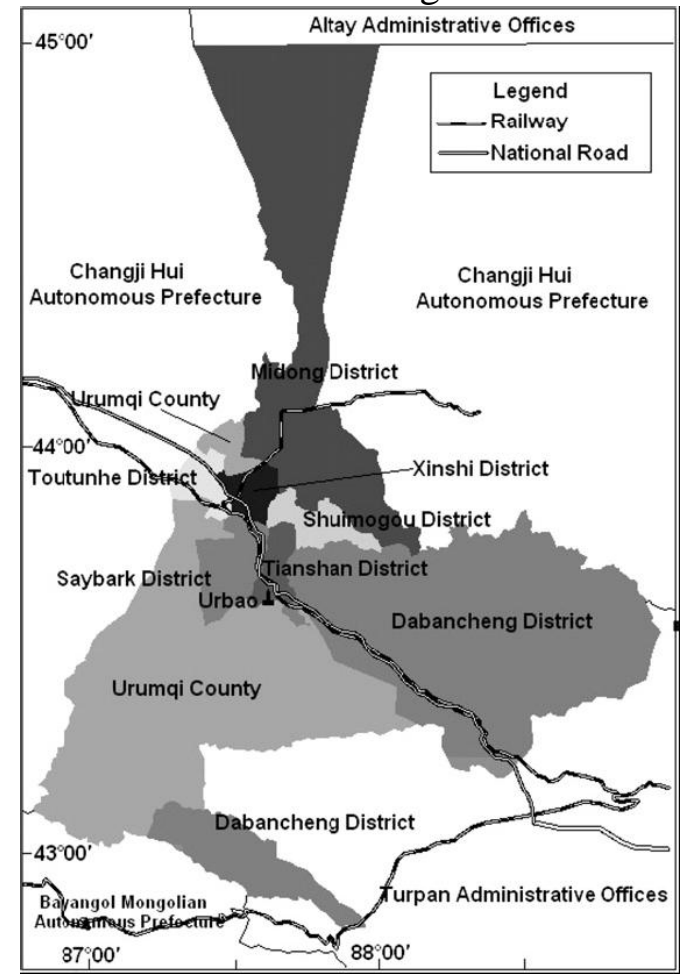

Fig. 1 District location and its vicinity area of Urumqi [6]

\section{SLEUTH Dynamic Urban Growth Model}

SLEUTH is a self-modifying probabilistic cellular automata model originally developed by Keith Clarke at the University of California at Santa Barbara. It's an initial abbreviation for the input data layers: Slope, Land Use, Exclusion, Urban, Transportation, and Hill Shade. The version used in this application, SLEUTH 3.0Beta, consists of an urban growth model (UGM) and an optional Land Cover Deltatron Model (LCDM). The UGM simulates urban growth, which is a non-urban cell will be converted to an urban cell. The LCDM, which is driven by the UGM, simulate land use and land cover change. In this application, only the urban module was utilized, so each cell in the study area had only two possible states: urbanized or non-urbanized. SLEUTH simulates four types of urban growth (Spontaneous growth, New spreading center growth, Edge growth, and Road-influenced growth) controlled through the interactions of five growth coefficients (ranging from 0 to 100): Dispersion, Breed, Spread, Road gravity, and Slope [7].

These growth rules are controlled by five growth parameters: diffusion, breed, spread, road gravity and slope resistance. Each parameter has a range of $0 \sim 100$ which is dimensionless and shows the importance 
of the corresponding parameter. Fig. 2 shows the relationship between the urban growth rules and the five growth parameters [8].

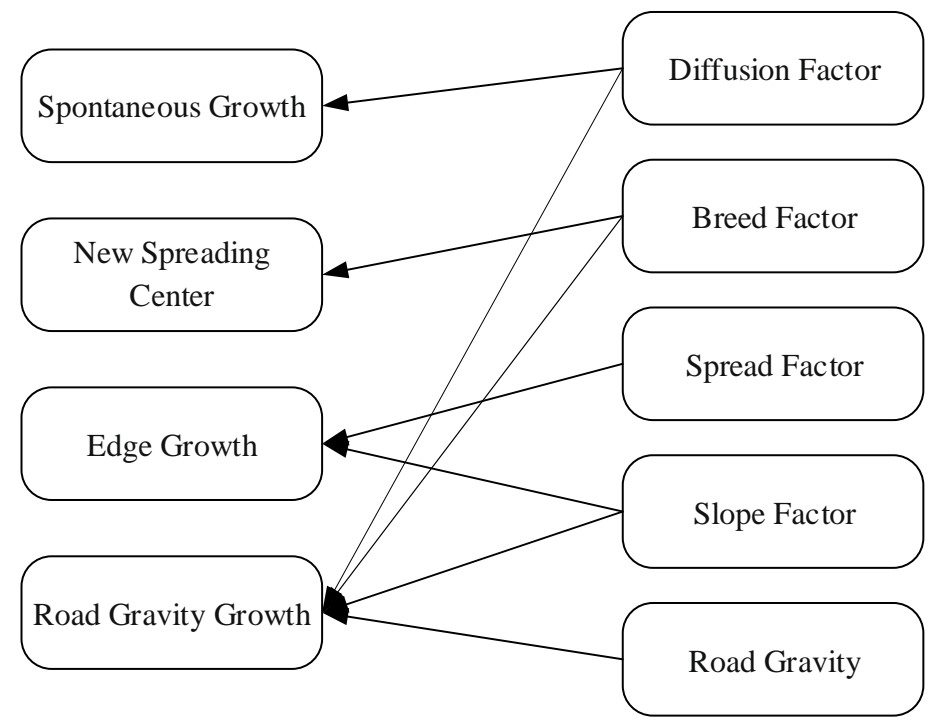

Fig. 2 The relationship between growth rules and urban growth parameters [8]

This paper documents the process of adapting and calibrating the SLEUTH model to the local characteristics of Urumqi. The purposes were: (1) to demonstrate that the same model could apply not only to central and eastern China but also to northwestern cities; (2) to demonstrate how important structural and geographical differences between applications could be revealed by the calibrations that may be of use in comparative urban study; (3) how a sequential multistage optimization throughout different phases of calibration is the key to model application; (4) provide the some reference for the city managers in the aspects of urban land use.

\section{Calibration of the SLEUTH Model}

For calibration, the SLEUTH requires inputs of historic urban extent for at least four time periods, a historic transportation network for at least two time periods, slope, and an excluded layer. Hereinto, the excluded layer designates lands that are resistant to urban development. For the excluded layer used in calibration, water bodies, parks, forests and other ecological preservation areas were entirely excluded from development. The excluded layer is typically scaled from 0 (no exclusion) to 100 (completely excluded).

Tab. 1 Best values for the parameters under different scenarios

\begin{tabular}{|c|c|c|c|c|}
\hline $\begin{array}{c}\text { Dispersion } \\
\text { Coefficient }\end{array}$ & Breed Coefficient & $\begin{array}{c}\text { Spread } \\
\text { Coefficient }\end{array}$ & Slope Coefficient & $\begin{array}{c}\text { Road Gravity } \\
\text { Coefficient }\end{array}$ \\
\hline 1 & 36 & 83 & 19 & 35 \\
\hline
\end{tabular}

A large number of combinations of parameter values are tested automatically and the user evaluates the results, locating a "best fit" set of parameter values through the use of fit statistics. We performed what is referred to as a coarse calibration, where the values for each parameter ranged from 1 100, but only increments of 25 were tested (i.e.1, 25, 50, 75, and 100). This resulted in 3125 unique parameter combinations. Than we performed fine calibration and final calibration orderly, and the values had narrow ranges and small increments. The fine calibration and final calibration resulted 5400 and 3240 unique parameter combinations respectively. Finally, we got the best values for the parameters as follows (Table 1).

\section{Forecasts to 2030}

When forecasts are created with SLEUTH, the model is initialized with the latest urban extent map, 
and the growth coefficient values that were derived during calibration. The user sets the target year in which to stop the forecast, and in our case we chose the year 2030. The model shows that the major growth modes before 2020 were mainly "Edge Growth" and slightly "Road-Influenced Growth". In another word, new developments tended to occur mostly at the edge of existing urban areas or circled around newly developed areas and main roads. From 2020 to 2030, besides "Edge Growth" and "Road-Influenced Growth" modes, "Spontaneous Growth" and "New Spreading Center Growth" occur and tend to increase. In details, Fig. 4 shows the observed pixels produced by "Edge Growth" (eg), "Road-Influenced Growth" (rg), "Spontaneous Growth" (sg) and "New Spreading Center Growth" (nscg). The result revealed the findings bellow. First, throughout the forecast period, we can sort the growth modes in order as follows: eg, rg, sg and nscg. Hereinto, eg index stands in an absolute dominant position. Second, eg index shows a long-time increasing trend, especially in the first 5 years. However, after that it grows at a relatively slower speed. Third, $\mathrm{rg}$ index declines on the whole, significantly before 2015. Finally, we find that, both of sg index and nscg index are quite stable throughout, which basically float between 1 and 4 .
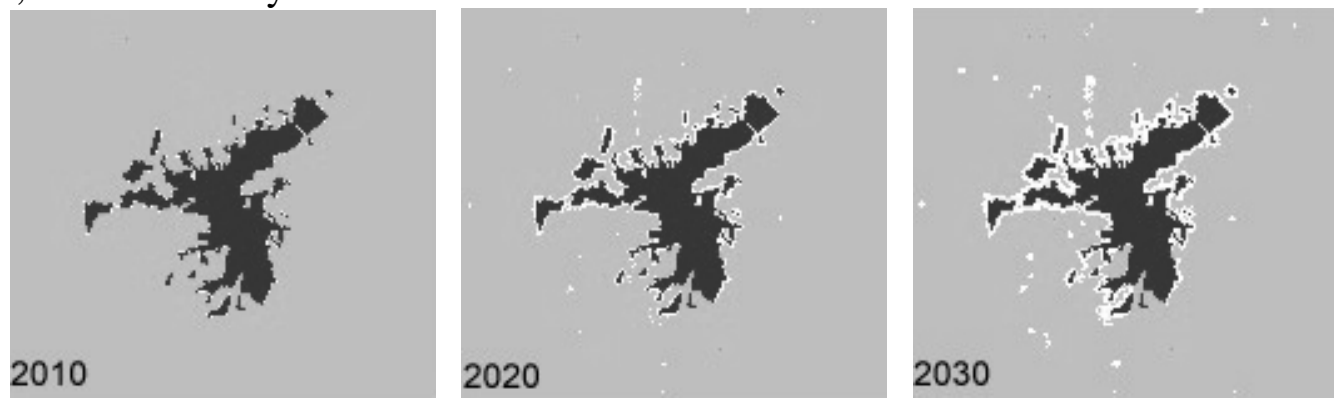

Fig. 3 The urban expansion maps of Urumqi

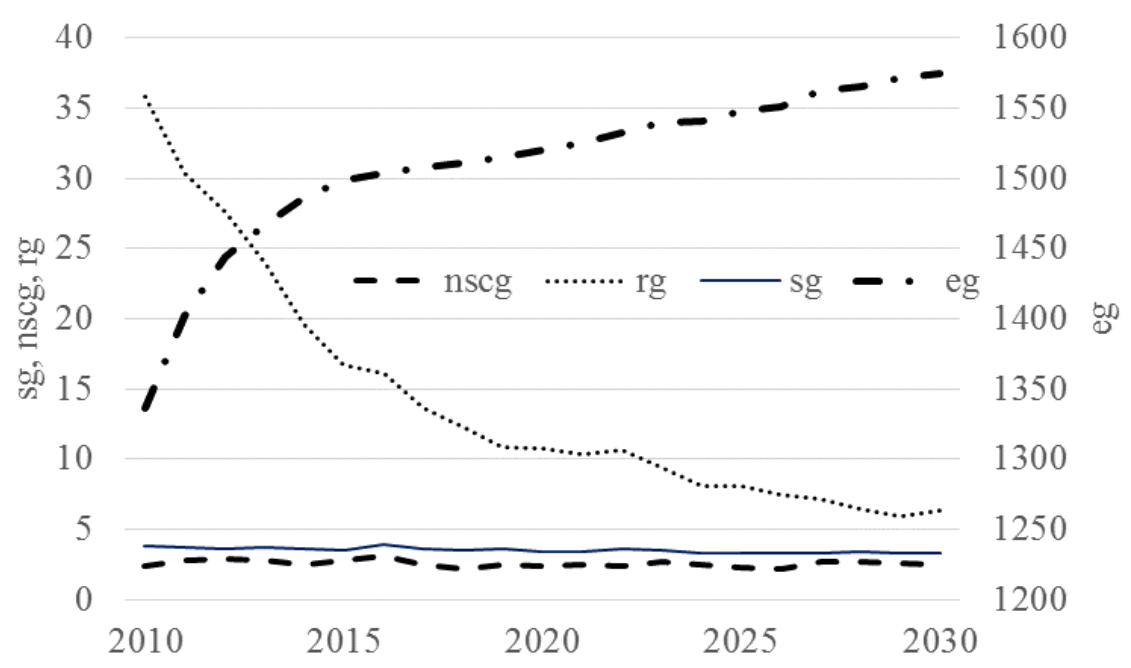

Fig. 4 The index variation of different growth type

From spatial aspect, the growth of northern, western and eastern directions is very obvious, and there exist the above four growth modes. After 2020, the "Edge Growth" in southern direction is apparently limited. This could be explained by the features of urban development in a mountain environment. Specifically, the Tianshan Mountains resist the urban expansion in southern direction.

\section{Conclusions and Discussion}

Urban growth throughout the world has led to consumption of land resources and degradation of vast land resources. In order to analyze and understand the land conversion in cities, models can be used as innovative tools to support spatial urban planning for sustainable development. This research employed SLEUTH model to simulate future urban growth in a northwestern city. On this basis, we analyzed the main growth modes and the overall tendency. However, the result is based on some basic hypothesis and fixed algorithm. It could not completely reflect the realistic situation and refer all of the factors. Because, 
we noted that urban growth is a complex process which is also affected by population increase, infrastructures and other socioeconomic factors. Unfortunately, the SLEUTH model only considers road networks of the set of infrastructure parameters possibly involved in urban expansion. Furthermore, in the case of Urumqi City, development is controlled not only by land use planning results, municipality decisions and sparse unharnessed development of built-up areas, but also others factors which are influential as well. However, the model is still effective and serves as a decision support tool and helps city managers realize the outcome of possible actions they might take.

Lastly, the results revealed some conclusion which were with realistic significance as follows. In the future years during the forecast period, Urumqi city should focus on enforcing the urban internal spatial structure optimization and spatial displacement, paying attention to the urban internal differentiation, improving the economic value and utilization of urban land resources, controlling the speed of urban growth, and retaining certain land reserve and development space for the future development.

\section{Acknowledgement}

This work was financially supported by the Natural Science Foundation of Xinjiang Uygur Autonomous Region (2012211B27).

\section{References}

[1] R. Rafiee, A. S. Mahiny, N. Khorasani, A. A. Darvishsefat and A. Danekar, Simulating urban growth in Mashad City, Iran through the SLEUTH model (UGM), Cities. 26 (2009) 19-26.

[2] P. Stalker, Handbook of World, Oxford University Press, New York, 2000.

[3] S. Leao, I. Bishop, D. Evans, Simulating urban growth in a developing nation's region using a CA-based model, Journal of Urban Planning and Development 130 (2004) 145-158.

[4] P. M. Torrens, How Cellular Models of Urban Systems Work, CASA Working Paper 28, University College London, Centre for Advanced Spatial Analysis (2000).

[5] K. C. Clarke, L. J. Gaydos, Loose-coupling a cellular automation model and GIS: Long-term urban growth prediction for San Francisco and Washington/Baltimore, International Journal of Geographical Information Science, 12 (1998) 699-714.

[6] W. Dong, X. L. Zhang, Urumqi, Cities, 28 (2011) 115-125.

[7] F. M. Xi, Y. M. Hu, H. S. He, X. Q. Wu, R. C. Bu, Y. Chang, M. Liu, J. Yu, Simulate urban growth based on RS, GIS, and SLEUTH model in Shenyang-Fushun metropolitan area northeastern China, in: 2009 Joint Urban Remote Sensing Event, Vols 1-3, pp. 959-968

[8] Y. C. Ding, Y. K. Zhang The simulation of urban growth applying SLEUTH CA model to the Yilan DELTA in Taiwan. Journal Alam Bina, 9 (2007) 95-106. 